\title{
HIGH PRECISION DTM AND DOM GENERATING USING MULTI-SOURCE ORBITAL DATA ON CHANG'E-4 LANDING SITE
}

\author{
B. Liu, S. Niu, X. Xin, M. Jia, K. Di *, Z. Liu, M. Peng, Z. Yue \\ State Key Laboratory of Remote Sensing Science, Institute of Remote Sensing and Digital Earth, Chinese Academy of Sciences, \\ Beijing, China - (liubin, xinxin, jiamn, dikc, liuzq, pengman, yuezy)@radi.ac.cn; niuvictory@163.com
}

Commission III, ICWG III/II

KEY WORDS: Chang'e-4 Landing Site, Digital Terrain Model, Digital Orthophoto Map, Multi-source Data, DTM Fusion, Lunar Orbital Imagery

\begin{abstract}
:
Chang'e-4 (CE-4) has successfully soft landed in Von Kármán crater inside the South Pole-Aitken (SPA) basin at 10:26 am on January 3, 2019. High precision landing site mapping plays an essential role in mission operations and science applications before and after landing. In this paper, we propose a novel method for generating the highest resolution and the best precision Digital Terrain Model (DTM) and Digital Orthophoto Map (DOM) of the CE-4 landing area using available multi-source data. First, the CE-2 DTM is co-registered to SLDEM2015. Then the vertical inconsistencies of the co-registered DTMs are analysed and the biases of CE-2 DTM with respect to SLDEM2015 are erased. Finally, a new DTM with more information and better precision is generated by fusion of the co-registered DTMs. Using the new DTM as a reference, a seamless DOM is generated based on block adjustment of projected imagery, which can reduce the inconsistencies among the corrected images to sub-pixel level. As a result, a DTM of the Von Kármán crater area $\left(42^{\circ} \mathrm{S}-48^{\circ} \mathrm{S} / 172{ }^{\circ} \mathrm{E}-180^{\circ} \mathrm{E}\right.$ ) and a seamless DOM covering an area of $2.3^{\circ}$ in longitude and $1^{\circ}$ in latitude with a ground sample distance of $0.9 \mathrm{~m}$ are generated using the developed method.
\end{abstract}

\section{INTRODUCTION}

Chang'e-4 (CE-4) has successfully soft landed in Von Kármán crater inside the South Pole-Aitken (SPA) basin at 10:26 am on January 3, 2019. The lander location is precisely determined to be $\left(177.588^{\circ} \mathrm{E}, 45.457^{\circ} \mathrm{S}\right)$ (Di et al., 2019). The rover was released from the lander and touched on the lunar surface on the same day. It is the first in-situ exploration on the far side of the moon by human spacecraft and is expected to gain new insights into the evolution of the moon (Meng et al., 2018). High precision landing site mapping plays an essential role in mission operations and science applications before and after landing. At present, the resolution of the available mosaic products covering CE-4 landing area can reach to $7 \mathrm{~m}$ /pixel (CE-2 CCD camera global mosaic) (http://moon.bao.ac.cn/) . SLDEM2015, a lunar DEM generated by co-registration and combining SELENE TC DEM with LRO laser altimetry data (Barker et al., 2016), and Chang'e-2 (CE-2) stereo images DTM (Li et al., 2018) are $\sim 42 \mathrm{~m} /$ pixel and $20 \mathrm{~m} /$ pixel respectively at CE-4 landing site. These maps are useful for analysis of the landing area, but may not be sufficient for detailed analysis and mission planning for their low resolutions.We are committed to producing the highest resolution and the best precision Digital Terrain Model (DTM) and Digital Orthophoto Map (DOM) of the CE-4 landing area using available multi-source data.

The laser altimeter data and the stereo image processing are two main sources for generating DTMs of the lunar surface. The Lunar Orbiter Laser Altimeter (LOLA) (Smith et al., 2009) DTM is widely used laser altimeter DTM of lunar surface. The highest resolution DTM interpolated from LOLA points is up to 1024 pixels/degree (about $30 \mathrm{~m}$ in the equatorial region). The laser altimeter data can achieve higher vertical precision but less planar density (thus less texture information in derived DTM). By contrast, the stereo image derived DTMs have more texture information but lower vertical precision. Thus it is possible and meaningful to combine two DTMs to produce a better DTM. It's worth to mention that SLDEM2015 is a usage case of this strategy. However, the DTM derived from different stereo images of the same area usually contains different information because of the different resolutions and illumination conditions of the images. The CE-2 DTM can also supply lots of supplement information to SLDEM2015.

The Lunar Reconnaissance Orbiter Camera (LROC) Narrow Angle Camera (NAC) has acquired highest resolution (up to 0.5 $\mathrm{m}$ ) images of nearly the entire moon (Robinson et al., 2010). Due to the precision limitation of lunar orbit and attitude measurements, the DOMs directly generated with the original rigorous geometric model usually have widespread inconsistencies. It is still highly demanded to produce large area seamless DOM using LROC NAC images (Di et al., 2018; Di et al., 2019).

In this paper, a new DTM with more information is produced by data fusion of CE-2 DTM and SLDEM2015. First, we coregistered the CE-2 DTM to SLDEM2015. Then we analysed the vertical inconsistencies of the co-registered DTMs and erase the biases of CE-2 with respect to SLDEM2015. Finally, a new DTM with more information and better precision was generated by fusion the processed DTMs. Using the new DTM as a reference, a seamless DOM is generated based on block adjustment of projected imagery.

\footnotetext{
* Corresponding author.
} 


\section{DATA}

The data sets used in this study include DTMs from CE-2 and SLDEM2015 and high resolution images from LROC NAC. The CE-2 DTM is generated by the CE-2 CCD stereo images and has a resolution of about $20 \mathrm{~m}$. The global CE-2 DTM product has been framed into 188 parts and each frame constitutes a data file (tif), a projection file (prj) and a header file (tfw) with same names. The CE-2 DTM products can be downloaded from the website of Data Publishing and Information Service System of China's Lunar Exploration Project (http://moon.bao.ac.cn/).

SLDEM2015 is a lunar DTM generated by co-registration and combining SELENE TC DTM with LRO laser altimetric data. It has a horizontal resolution of 512 pixels per degree ( $\sim 0 \mathrm{~m}$ at the equator and $\sim 42 \mathrm{~m}$ at CE-4 landing site) and an elevation precision of 3 - $4 \mathrm{~m}$ (Barker et al., 2016). The SLDEM2015 can be obtained from the Planetary Data System (http://pdsgeosciences.wustl.edu/).

The LROC NACs are important payloads of the LRO mission. It provides panchromatic images of the lunar surface with a pixel scale of $0.50 \mathrm{~m}$ from a $50 \mathrm{~km}$ altitude (Robinson et al., 2010), which are the highest-resolution lunar orbital images so far. The NAC images can also be downloaded from the PDS website.

\section{METHODOLOGY}

The scheme can be divided into two parts, the fusion DTM generation and the seamless DOM making. Figure 1 shows the flowchart of the large area DTM and DOM generation process.

In order to effectively eliminate the geometric deviation between different DTM products and make the comparison and the fusion of multi-source DTMs possible, a DTM matching method based on the simulated image was proposed. Firstly, the DTMs were transformed into an unified projection coordinate system. Then image simulation was carried out by converting DTMs to images. The produced simulation image was used for 2D matching to obtain the tie points between DTMs. In this paper, the Scale-Invariant Feature Transform (SIFT) (Lowe., 2004) algorithm was adopted. RANSAC algorithm was used to eliminate the gross errors, after which the transformation model was obtained by the matching points. CE-2 DTM was coregistrated to the SLDEM2015 using the transformation model. Through frequency domain transformation and analysis, the periodic system errors of CE-2 DTM has been removed. The frequency domain fusion was performed to generated the fusion DTM.

The multiple coverage LROC NAC images were selected by the principles which we discussed in the previous studies (Liu et al., 2018; Di et al., 2019) to produce the seamless DOM. The rigorous sensor model (RSM) of LROC NACs was established with exterior orientation (EO) and interior orientation (IO) parameters, which are retrieved from the relevant SPICE kernels (NAIF, 2014; Henriksen et al., 2016). The rational function model (RFM) were constructed by fitting the RSM with residual of 1/100-pixel level (Liu et al., 2016). Then using a coregistration method constrained by both geometric and photometric information (Xin et al., 2018), The LROC NACs were co-registration to the fusion DTM. We rectified the LROC NAC images to a given projection. But the geometric inconsistencies still exist for the precision limitation of the reference DTM. So we proposed a block adjustment method for projected images. After block adjustment, the DOMs were resampled and mosaicked.

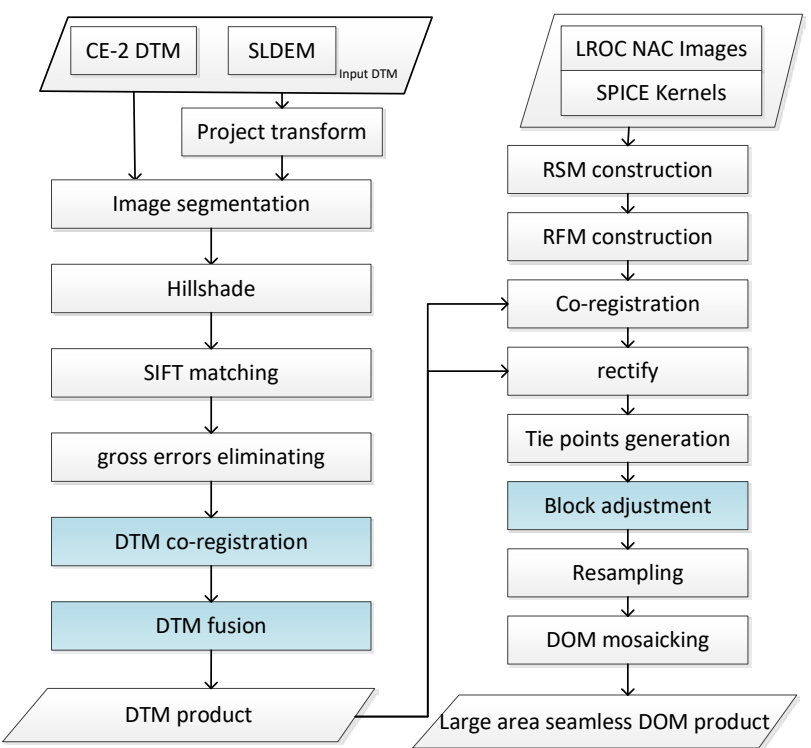

Figure 1. Flowchart of DTM fusion and DOM generation

\subsection{DTM co-registration and fusion}

The geometric positions and elevations of different DTMs are inconsistent. In order to get better comparative analysis and fusion of multi-source DTMs, these DTMs need to be matched to each other. Feature-based matching, which is to extract the feature points, feature lines and feature surfaces on DTM and match them with these features, is common used in previous studies (Kim et al., 2000; Wu et al., 2013). However, this method may fail in places with poor texture. In this paper, the simulation images of DTM are used for converting the 3D matching to 2D images matching. The planetary surface, such as the lunar surface has no artificial features and is mostly covered by the regolith. Thus, using the hill-shading method can produce a good image simulation effect. We also segmented the DTM to $1^{\circ} \times 1^{\circ}$ for parallel matching calculation.

DTMs are fusion in frequency domain based on Discrete Fourier Transformation (DFT, Bracewell, 2000). In order to extract the optimal information from different DTMs and fuse them to a high-precision DTM, The DTMs are converted into the frequency domain through DFT as displayed in Eq. (1).

$\mathrm{F}(\mathrm{u}, \mathrm{v})=\sum_{x=0}^{M-1} \sum_{y=0}^{N-1} f(x, y) e^{-j 2 \pi(u x / M+v y / N)}$

where $f(x, y)$ is the function of the DTM, M, N are sample and line of the DTM, u, v are the frequency variables of F(u,v) and $\mathrm{x}, \mathrm{y}$ are spatial variables of $f(x, y)$.

The inverse DFT (IDFT) is shown in Eq. (2).

$\mathrm{f}(\mathrm{u}, \mathrm{v})=\sum_{x=0}^{M-1} \sum_{y=0}^{N-1} F(x, y) e^{j 2 \pi(u x / M+v y / N)}$

According to our research, there is a periodic system error existing on Chang'E-2 DTM. The low frequency periodic noise is removed through a high-pass filter operation which is represented by the following equation. 


$$
\begin{aligned}
& \mathrm{g}(\mathrm{x}, \mathrm{y})=\mathfrak{J}^{-1}[\mathrm{H}(\mathrm{u}, \mathrm{v}) \mathrm{F}(\mathrm{u}, \mathrm{v})] \\
& \mathrm{H}(\mathrm{u}, \mathrm{v})= \begin{cases}0, & D(u, v) \leq D_{0} \\
1, & D(u, v)>D_{0}\end{cases}
\end{aligned}
$$

where $\mathfrak{\Im}^{-1}$ is IDFT, $\mathrm{g}(\mathrm{x}, \mathrm{y})$ is the noiseless DTM, $\mathrm{H}(\mathrm{u}, \mathrm{v})$ is high-pass filter, $\mathrm{F}(\mathrm{u}, \mathrm{v})$ is DFT result of original DTM, $D(u, v)$ is the distance from point $(\mathrm{u}, \mathrm{v})$ to the centre of the filter and $D_{0}$ is cut-off frequency.

Chang'E-2 DTM which is removed noise and SLDEM2015 which is up-resampled to the resolution of Chang'E-2 DTM are used to fuse a new high-precision DTM. The low frequency information of both of the two DTMs is extracted based on a low-pass filter. And the average low frequency information of the DTMs is generated as the low frequency information of the new DTM. Then a high-pass filter with the same cut-off frequency $D_{0}$ is used to extract the high frequency information of Chang'E-2 DTM. Finally, the high and low frequency information are summed, and the fused DTM is converted into the spatial domain through IDFT.

\subsection{Block Adjustment of Projected Imagery}

To deal with the geometric deviations between NAC images and SLDEM2015, this study proposed a novel block adjustment method based on the projected imagery. A proper map projection is applied to all the images with the refined geometric models obtained by last step and the projected images can be generated.

A novel block adjustment method for the projected images is proposed in this study to improve the geometric consistencies between images. With the matched tie points between the projected images, the corresponding projected coordinates are calculated. The affine transformation model is used in the block adjustment and the error equations can be established as Eq. (4).

$$
\begin{aligned}
& v_{x i}^{j}=D x_{i}^{j^{\prime}}-D x_{i}=a_{0}^{j}+a_{1}^{j} D x_{i}^{j}+a_{2}^{j} D y_{i}^{j}-D x_{i} \\
& v_{y i}^{j}=D y_{i}^{j^{\prime}}-D y_{i}=b_{0}^{j}+b_{1}^{j} D x_{i}^{j}+b_{2}^{j} D y_{i}^{j}-D y_{i}
\end{aligned}
$$

where, $\mathrm{i}$ and $\mathrm{j}$ are the image IDs, $D x_{i}^{j}, D y_{i}^{j}$ and $D x^{j^{\prime}}, D y_{i}^{j^{\prime}}$ are projected coordinates before and after block adjustment, respectively, $a_{0}^{j}, a_{1}^{j}, a_{2}^{j}, b_{0}^{j}, b_{1}^{j}, b_{2}^{j}$ are affine transformation parameters.

By solving the error equations with the least squares method, the affine transformation parameters can be obtained. Then, the affine transformation is applied to each projected image and the geometric inconsistencies between the images can be reduced.

\section{RESULTS}

\subsection{DTM co-registration results}

Based on the given simulated illumination information, the shaded relief maps of SLDEM2015 and CE-2 DTM are generated. Figure 2 displays the two shaded relief maps in Von Kármán crater. The two shaded relief maps have similar surface features which make it possible to match the DTMs through two-dimension feature match method (e.g. SIFT). The gross errors were eliminated through RANSAC algorithm, and 684 tie points were extracted from the DTMs. Using the gross errors eliminated matching points, a second-order polynomial transformation model was calculated. And CE-2 DTM is coregistrated using the parameters.

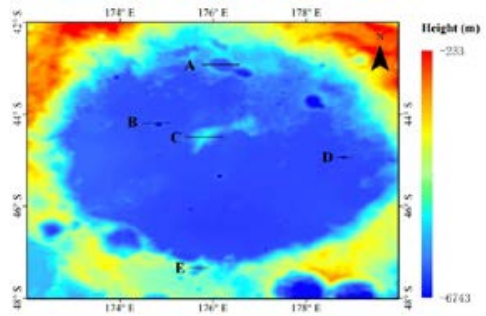

(a) SLDEM2015

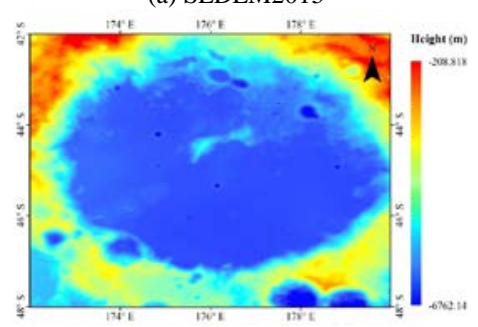

(c) CE-2 DTM

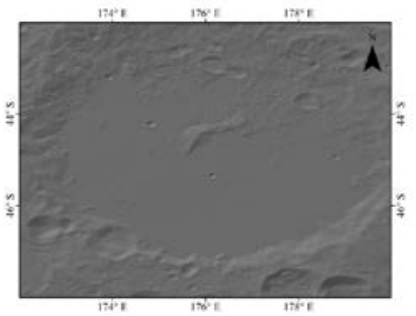

(b) Shaded relief map of SLDEM2015

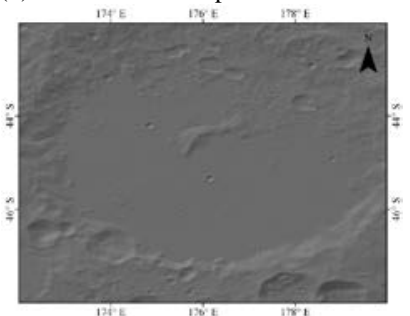

(d) Shaded relief map of CE-2 DTM
Figure 2. The DTMs and shaded relief maps

The original DTM of CE-2 and the registered DTM of CE-2 were subtracted from SLDEM2015 pixel by pixel, respectively. Figure 3 (a) shows that the height different between original CE-2 DTM and SLDEM2015 are widely distributed. most of the difference are bigger than $200 \mathrm{~m}$ among the craters. (b) shows that after matching the differences are mostly in the range of $-100-100 \mathrm{~m}$. The influence of plane difference on the height difference is more significant where the height difference is large (such as the edge of the crater). Before registration, the elevation difference between the two DTMs is significant in these places. This phenomenon is not obvious after the registration, which indicates that the plane position becomes more accurate because of the registration. The statistical results in Table 1 also supports this point.

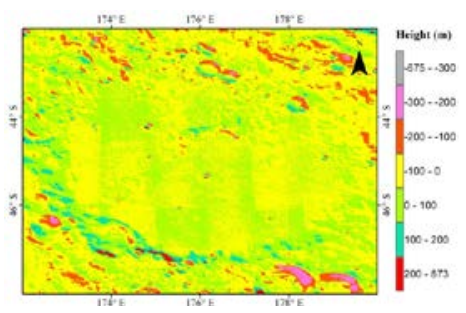

(a) Original CE-2 DTM

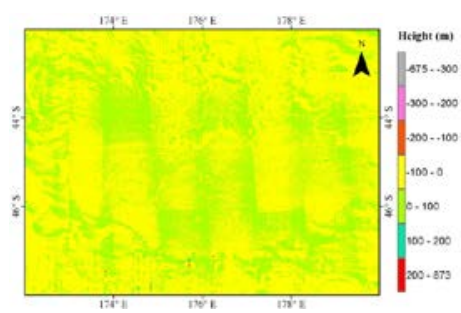

(b) Co-registrated CE-2 DTM
Figure 3. Differences between CE-2 DTM and SLDEM2015

Table 1. Height difference between SLDEM2015 and CE-2 DTMs (original DTM and co-registrated DTM).

\begin{tabular}{ccc}
\hline $\begin{array}{c}\text { Difference with } \\
\text { SLDEM2015 }\end{array}$ & $\begin{array}{c}\text { Average value } \\
\text { (m) }\end{array}$ & $\begin{array}{c}\text { Standard } \\
\text { deviation }(\mathbf{m})\end{array}$ \\
\hline Original CE-2 & -11.52 & 55.55 \\
Co-registrated CE-2 & -7.24 & 11.90 \\
\hline
\end{tabular}

Some detailed analysis was made by compare the height profile of some craters. The results in Figure 4 shows that after coregistrated, matched CE-2 DTM is more consistent with the SLDEM2015.

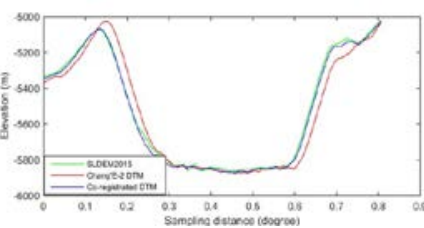

A in Figure 2(a)

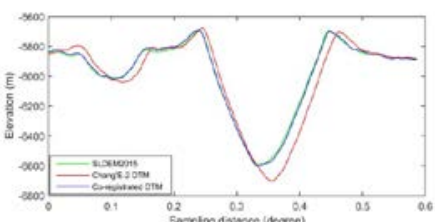

$B$ in Figure 2(a) 


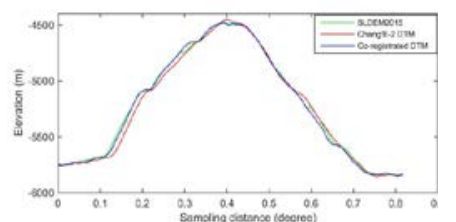

$\mathrm{C}$ in Figure 2(a)

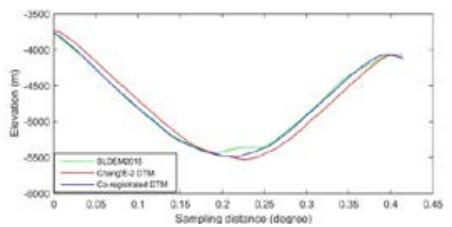

E in Figure 2(a)

Figure 4. Comparision of height profiles

\subsection{DTM fusion Results}

Though the CE-2 DTM has been co-registrated to SLDEM2015, the difference of the height between them was still too large to fusion. From Figure 3 (b), we found that periodic gross errors of vertical direction exist in CE-2 DTM. Using Fourier Transformation and filter operation, the periodic gross errors extracted from CE-2 DTM (Figure 5). There are clearly stripes oriented in perpendicular direction of the orbiter track. Figure 6 displays the profiles of $\mathrm{A}$ and $\mathrm{B}$ in Figure 5. The width of the strip are $\sim 21 \mathrm{~km}$. Along the track, some periodic errors also can be found. These errors may be introduced thought the data procedure.

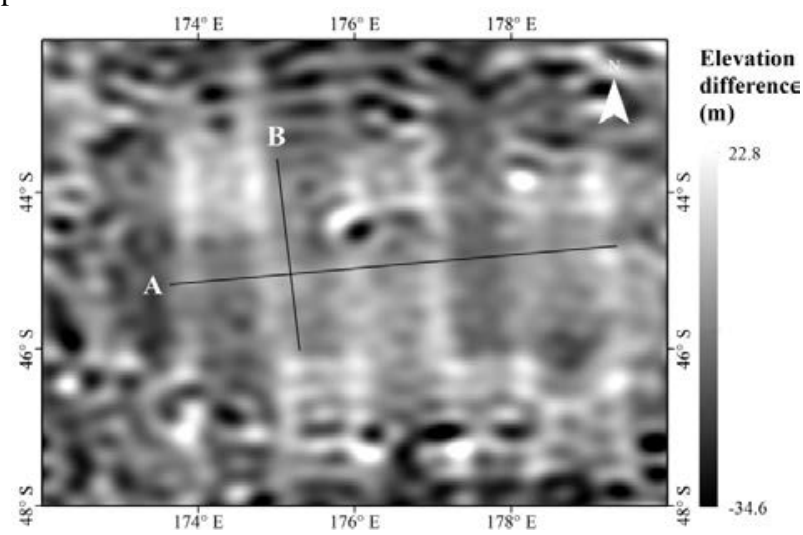

Figure 5. Periodic gross errors extracted from CE-2 DTM.

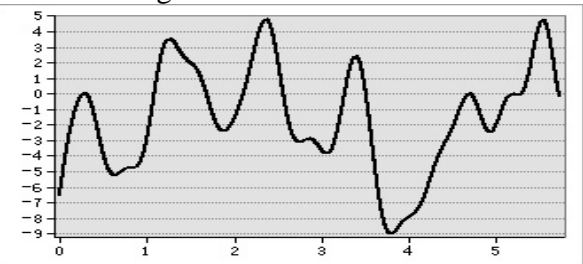

(a) Profile A of Fig. 5.

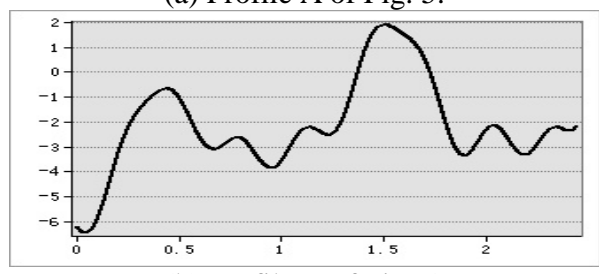

(b) Profile B of Fig. 5.

Figure 6. Profile analysis in Figure 5.

After eliminating the gross errors of CE-2 DTM, a new DTM that combined the CE-2 and SLDEM2015 has been produced via frequency domain fusion method proposed in this paper. Figure 7 is the fusion DTM.

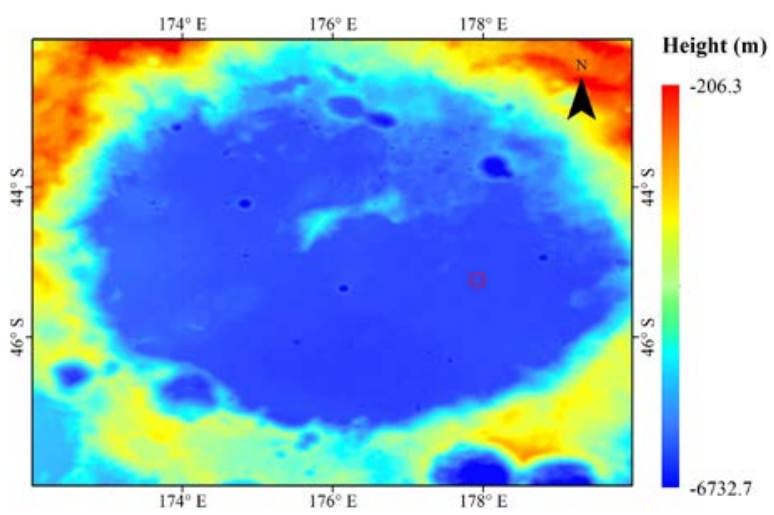

Figure 7. Fused DTM

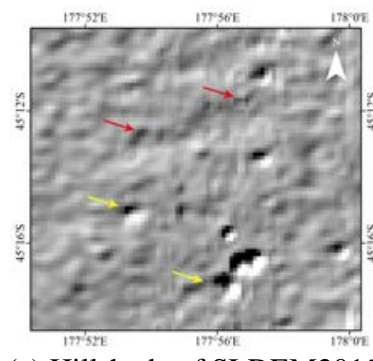

(a) Hillshade of SLDEM2015

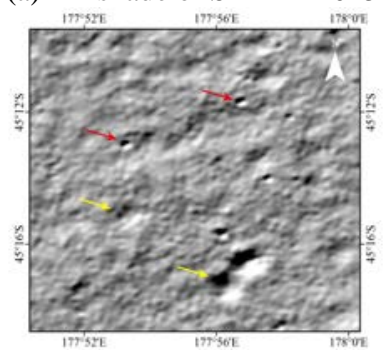

(c) Hillshade of fused DTM

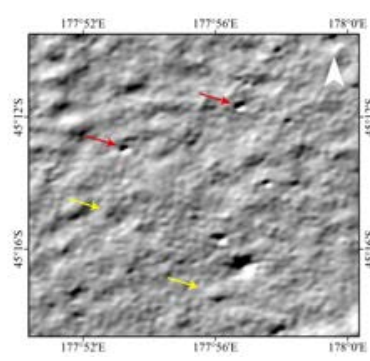

(b) Hillshade of CE-2 DTM

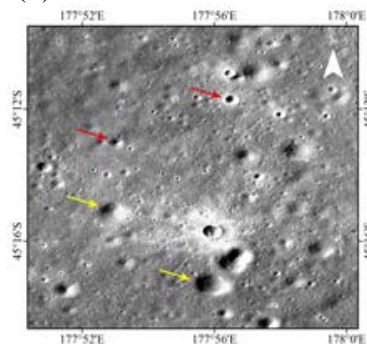

(d) LROC NAC image
Figure 8. Comparisons of the DTMs in local area. The red arrows indicate the details only in CE-2 DTM. The yellow arrows indicate the details only in the SLEDM2015

Using the shade map to evaluate our method, we found the new fusion DTM contains more information than both the CE-2 DTM and SLDEM2015.

\subsection{Seamless DOM Generation}

One hundred LROC NAC images were selected to generated the DOM. For the lacking coverage of LROC NAC images, the $7 \mathrm{~m} /$ pixel CE-2 DOM also used in some area for gap filling. The seamless DOM (Figure 9) covers an area of $2.3^{\circ}$ in longitude and $1^{\circ}$ in latitude, with a ground sample distance of $0.9 \mathrm{~m}$. 


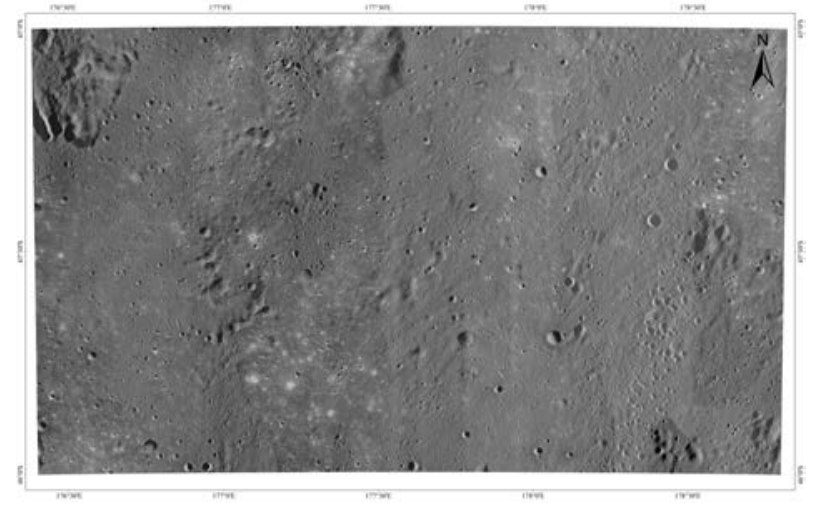

Figure 9. The resultant seamless DOM mosaic of CE-4 landing area.

\section{CONCLUSIONS}

This paper presented a novel scheme for generating seamless DTM and DOM using multi-source orbital data. A fused DTM of the Von Kármán crater area $\left(42^{\circ} \mathrm{S}-48^{\circ} \mathrm{S} / 172{ }^{\circ} \mathrm{E}-180^{\circ} \mathrm{E}\right)$ was generated by fusion of CE-2 DTM and SLDEM2015. The DTM fusion results show that the fused DTM contains more information than both SLDEM2015 and CE-2 DTM. We also produced a seamless DOM covers the CE-4 landing site with a ground sample distance of $0.9 \mathrm{~m}$. The DOM and DTM have been used in several tasks, e.g., CE-4 lander localization, topographic analysis of the landing site, sky (relay satellite) visibility analysis, etc., and will continue to be used along with lander and rover images for further science applications.

\section{ACKNOWLEDGEMENTS}

This work was supported by the National Natural Science Foundation of China (Grant No. 41671458, 41590851, and 41771490) and the Key Research Program of the Chinese Academy of Sciences (NO. XDPB11). The authors thank all those who worked on the Planetary Data System archive to make the LROC imagery and SLDEM2015 available, the Lunar and Deep Space Exploration Science Applications Center of the National Astronomical Observatories for providing the Chang'e-2 DOM and DEM.

\section{REFERENCES}

Barker, M.K., Mazarico, E., Neumann, G.A., Zuber, M.T., Haru yama, J., Smith, D.E., 2016. A new lunar digital elevation mode 1 from the Lunar Orbiter Laser Altimeter and SELENE Terrain Camera. Icarus, 273, pp.346-355.

Bracewell, R. N., 2000. The Fourier Transform and its Applications, 3rd ed. McGrawHill, New York.

Di, K., Jia, M., Xin, X., Liu, B., Liu, Z., Peng, M., Yue, Z., 2018. High resolution seamless DOM generation over Chang'e5 landing area using LROC NAC images, 2018 ISPRS TC III Mid-term Symposium "Developments, Technologies and Applications in Remote Sensing”, 7-10 May, Beijing, China, pp. 271-276.

Di, K., Jia, M., Xin, X., Wang, J., Liu, B., et al., 2019. Highresolution large-area digital orthophoto map generation using
LROC NAC images. Photogrammetric Engineering \& Remote Sensing. (Accepted)

Di, K., Liu, Z., Liu, B., Wan, W., Peng, M., Wang, Y., Gou, S., Yue, Z., Xin, X., Jia, M. and Niu, S., 2019. Chang'e- 4 lander localization based on multi-source data. Journal of Remote Sensing, 23(1), pp.177-184.'

Henriksen, M.R., Manheim, M.R., K.N.Burns, Seymour, P., Speyerer, E.J., Deran, A., Boyd, A.K., Howington-Kraus, E., Rosiek, M.R., Archinal, B.A., Robinson, M.S., 2016. Extracting Accurate and Precise Topography from LROC Narrow Angle Camera Stereo Observations. Icarus, 283, pp.122-137.

Kim, J.R., Muller, J.P. and Morley, J., 2000. Automated MOLA track registration in MOC and Viking images and it's application for the establishment of new 3-D control points on Mars, International Archives of Photogrammetry and Remote Sensing, Amsterdam, pp. 469-475.

Li, C., Liu, J., Ren, X., et al., 2018. Lunar global high-precision terrain reconstruction based on chang'e-2 stereo images. Geomatics \& Information Science of Wuhan University, 43(4), pp.485-495.

Liu, B., Jia, M., Di, K., Oberst, J., Xu, B., Wan, W., 2018. Geopositioning precision analysis of multiple image triangulation using LROC NAC lunar images, Planetary and Space Science, 162, pp.20-30.

Liu, B., Xu, B., Di, K., Jia, M., 2016. A Solution to Low RFM Fitting Precision of Planetary Orbiter Images Caused by Exposure Time Changing. International Archives of the Photogrammetry, Remote Sensing and Spatial Information Sciences, XLI-B4, pp. 441-448.

Lowe, D.G., 2004. Distinctive Image Features from ScaleInvariant Keypoints. International Journal of Computer Vision. 60, pp.91-110.

Meng, Z., Li, C., Ping J., et al, 2018. Analysis about the landing site selection and prospective scientific objectives of the Von Kármán crater in Moon farside. Journal of Deep Space Exploration, 5(1), pp.3-11.

NAIF, 2014. Lunar Reconnaissance Orbiter Camera (LROC) Instrument Kernel v18. http://naif.jpl.nasa.gov/pub /naif/pds/ data/lro-l-spice-6-v1.0/lrosp_1000.

Wu, B., Guo, J., Hu, H., Li, Z., and Chen Y., 2013. CoRegistration of lunar topographic models derived from Chang'E-1, SELENE, and LRO laser altimeter data based on a novel surface matching method. Earth and Planetary Science Letters, 364, 68-84.

Xin, X., Liu, B., Di, K., Jia, M., Oberst, J., 2018. Highprecision co-registration of orbiter imagery and digital elevation model constrained by both geometric and photometric information. ISPRS Journal of Photogrammetry and Remote Sensing, 144(2018), pp.28-37. 